\title{
Identification of neprilysin as a potential target of arteannuin using computational drug repositioning
}

\author{
Xuan-Yi Ye', Qing-Zhi Ling², Shao-Jun Chen ${ }^{2, *}$ \\ ${ }^{1}$ College of Ecology, Lishui University, Lishui, China, ${ }^{2}$ Department of Traditional Chinese Medicine, Zhejiang Pharmaceutical \\ College, Ningbo, China
}

\begin{abstract}
The discovery of arteannuin (qinghaosu) in the $20^{\text {th }}$ Century was a major advance for medicine. Besides functioning as a malaria therapy, arteannuin is a pharmacological agent in a range of other diseases, but its mechanism of action remains obscure. In this study, the reverse docking server PharmMapper was used to identify potential targets of arteannuin. The results were checked using the chemical-protein interactome servers DRAR-CPI and DDI-CPI, and verified by AutoDock Vina. The results showed that neprilysin (also known as CD10), a common acute lymphoblastic leukaemia antigen, was the top diseaserelated target of arteannuin. The chemical-protein interactome and docking results agreed with those of PharmMapper, further implicating neprilysin as a potential target. Although experimental verification is required, this study provides guidance for future pharmacological investigations into novel clinical applications for arteannuin.
\end{abstract}

Uniterms: Arteannuin/chemical-protein interactome. Computational drug repositioning. Neprilysin/ identification. Reverse docking.

\section{INTRODUCTION}

Arteannuin (Figure 1) was originally discovered as a malaria therapy by Chinese scientists in the 1970s, and this discovery constitutes one of the greatest discoveries in medicine in the $20^{\text {th }}$ Century (O'Neill, Barton Ward, 2010; Tu, 2011). Arteannuin is also known as artemisinin, artemisine, Qinghaosu, and Huanghuasu (O'Neill et al., 2010; Tu, 2011). Artemisinin and its derivatives are unique among antimalarial drugs because they kill the young intraerythrocytic malaria parasite, thereby preventing its advancement into a more pathological mature stage (White, 2008). More recently, anticancer properties in cell lines and animal models have been reported, as has anti-schistosomal activity (O'Neill et al., 2010). Five key signalling pathways (PI3K-Akt, T-cell receptor, Toll-like receptor, TGF- $\beta$, and insulin) are reportedly involved in arteannunin-mediated anticancer effects (Huang et al., 2013), but the underlying mechanism of action remains ambiguous (O’Neill et al., 2010).

\footnotetext{
*Correspondence: S.-J. Chen. Department of Traditional Chinese Medicine. Zhejiang Pharmaceutical College. Ningbo 315100, China. E-mail: chenshaojun@hotmail.com
}

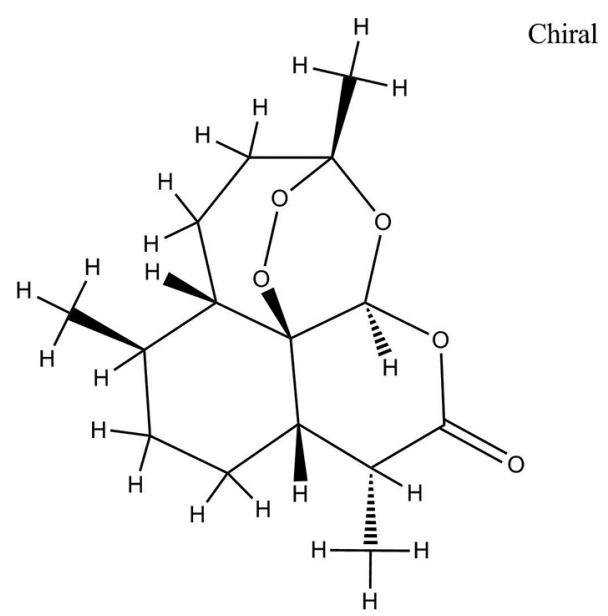

FIGURE 1 - The structure of arteannuin downloaded from PubChem (CID: 68827).

Drug discovery is a cost- and time-consuming venture (Hurle et al., 2013; Volkamer, Rarey, 2014). To reduce these expenses, computational approaches have been applied to the early drug-development pipeline (Volkamer, Rarey, 2014). In silico drug target identification, which involves numerous distinct algorithms for identifying disease-associated genes and 
proteins, is the first step in the drug discovery pipeline (Liu et al., 2010). Reverse docking, first proposed in 2001, refers to the computational docking of a specific small molecule of interest (natural product, lead, or synthetic compound) to a protein structure database (Chen, Zhi, 2001). This approach can be used to discover new targets for existing drugs and natural compounds, investigate the polypharmcology and molecular mechanism of a substance, find alternative drug information through drug repositioning, and predict adverse drug reactions and drug toxicity (Aeri, Kyoungyeul, Dongsup, 2016). This computational method is also particularly useful for identifying targets for a given ligand (Aeri, Kyoungyeul, Dongsup, 2016). In our previous work, we use reverse docking to reveal that carbonic anhydrase 2 may be a potential target of capsaicin, and other research groups have also identified capsaicin as a potent inhibitor of carbonic anhydrase isoenzymes (Ye, Ling, Chen, 2015). Adoption of computational methodologies for drug repositioning is rapidly becoming mainstream (Hurle et al., 2013).

In this study, we used computational drug repositioning to identify potential targets of arteannuin. The reverse docking server PharmMapper was used to identify potential targets, and the results were checked by AutoDock Vina in PyRx0.8, and the chemical-protein interactome servers DRAR-CPI and DDI-CPI. The ultimate aim of the study was to gain insight into the mechanism of arteannuin in traditional Chinese medicine.

\section{MATERIALS AND METHODS}

\section{Target prediction by PharmMapper}

PharmMapper (http://59.78.96.61/pharmmapper) is a web server for identification of potential drug targets that utilises a large-scale reverse pharmacophore mapping strategy (Liu et al., 2010). The server hosts a large, inhouse repertoire of pharmacophore database information annotated from TargetBank, BindingDB, DrugBank, and other potential drug target databases, and includes over 7000 receptor-based pharmacophore models (Liu et al., 2010). Recently, it was used to identify anticancer targets from eco-friendly waste generated from the peelings of Punica granatum (Usha et al., 2014), and also to predict and evaluate anticancer targets from cryptotanshinone (Yuan et al., 2014). In our previous work, we used the server to predict potential targets for Danshensu and Tanshinone IIA (Chen, 2014; Chen, Ren, 2014). PharmMapper is therefore a powerful tool for target prediction.
As described previously (Chen, 2014; Chen, Ren, 2014), a 2D SDF file for arteannuin was downloaded from the PubChem database (CID: 68827) and converted into the mol2 format using Discovery Studio Visualizer 3.5. The mol2 file was then submitted to the PharmMapper server, with the maximum generated conformation set at 300 , all targets set at 7302 , and the number of reserved matched targets set at 300 . Default values were used for all other parameters.

\section{Prediction of the chemical-protein interactome using DRAR-CPI and DDI-CPI}

DRAR-CPI, freely available at http://cpi.bio-x. $\mathrm{cn} / \mathrm{drar} /$, is a server for identifying drug repositioning potential and adverse drug reactions via the chemicalprotein interactome (Luo et al., 2011). The natural compound plumbagin was recently predicted to interact with 78 proteins involved in cell proliferation and apoptosis, immunity, and signal transduction following bioinformatics analysis that included DRAR-CPI (Qiu et al., 2015). We have also used the server previously to assess protein targets predicted by the PharmMapper server (Chen, 2014). All available evidence suggests that DRAR-CPI is a powerful computational tool for drug repositioning.

DDI-CPI (freely accessible at http://cpi.bio-X.cn/ $\mathrm{ddi} /$ ) is another server that predicts drug-drug interactions through implementing a chemical-protein interactome (Luo et al., 2014). It can predict the potential usefulness of a given compound among 2515 drug molecules in the library ( $85 \%$ of which are FDA-approved) (Luo et al., 2014). DDI-CPI can therefore be used to check the results of reverse docking.

As described previously (Chen, 2014; Qiu et al., 2015), the 2D structure of arteannuin was downloaded from PubChem, and hydrogens and Gasteiger charges were added and the file format converted to Mol2 using VegaZZ. The resultant file was uploaded to the DRARCPI and DDI-CPI servers, with default values for all parameters.

\section{Molecular docking using AutoDock Vina in PyRx 0.8}

AutoDock Vina in PyRx0.8 is a virtual screening software for computational drug discovery that can be used to screen libraries of compounds against potential drug targets (Trott, Olson, 2010). Recently, this software was used for the virtual screening of potential inhibitors of high-risk human papillomavirus 16 E6 protein (Kumar 
et al., 2015), and was also applied in research on ecofriendly waste from Punica granatum (Usha et al., 2014).

A redocking study using AutoDock Vina 1.0.2 was previously reported (Trott, Olson, 2010) that used a protein-ligand structure of a target protein (neprilysininhibitor complex, PDB code 1R1H) derived from the RCSB Protein Data Bank. The coordinates for the target protein and inhibitor bound to chain A were extracted and saved in separate files, and receptor and ligand input files were prepared using AutoDock Tools 1.5.6 (The Scripps Research Institute, CA, USA) (Morris et al., 2009) via the graphical user interface. The search space was restricted to a box of $24.965 \times 22.469 \times 22.780 \AA$ (centre $=31.883$, $44.050,34.157)$, which was centred on the ligand-binding site in the co-crystalised structure. Default values were used for all parameters. After docking, the highest scoring pose was selected for detailed analysis, and AutoDock Vina was used to dock the arteannuin-target protein complex.

\section{RESULTS}

\section{Targets predicted by PharmMapper}

The output of PharmMapper ranks potential target pharmacophore models in descending order based on fit score (Liu et al., 2010). The top ten disease-related targets identified in this study (Table I) revealed neprilysin as the highest hit. The neprilysin pharmacophore has three hydrophobic groups, one positive group, one negative group, two hydrogen donors, and three hydrogen acceptors (Figure 2). Pharmacophore visualisations are shown in Figure 2.
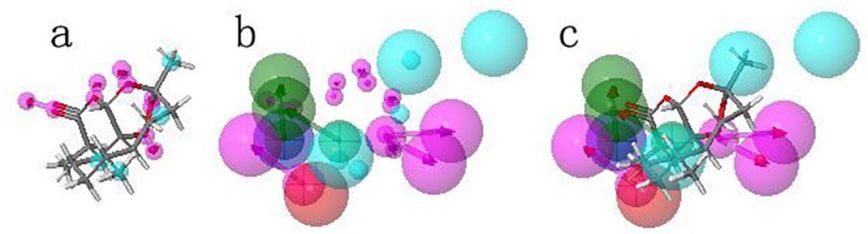

FIGURE 2 - Alignment of arteannuin and the pharmacophore model of neprilysin. (a) Arteannuin features. (b) Pharmacophore model. (c) Superimposition of the models in (a) and (b). Note: Pharmacophore features are coloured cyan for hydrophobic, blue for positively charged, red for negatively charged, green for hydrogen bond donor, and magenta for hydrogen bond acceptor (Liu et al., 2010).

\section{Prediction of the chemical-protein interactome by DRAR-CPI and DDI-CPI}

The affinity score, Z-score, and Z'-score of arteannuin-neprilysin obtained form the chemicalprotein interactome are shown in Table II, and the binding pattern obtained from the DRAR-CPI server is shown in Figure 3. The docking score of arteannuin-neprilysin obtained using the DDI-CPI server was $-7.5 \mathrm{kcal} / \mathrm{mol}$ (Table II).

\section{Molecular docking}

As illustrated in Figure 4, the top scoring redocking pose is very close to the ligand conformation observed in the co-crystal structure, with a root mean square deviation (RMSD) of only $1.12 \AA$ for non-hydrogen atoms. The redocking results indicated that the docking procedure can generate a physiologically relevant pose that is suitable for further studies.

TABLE I - Top ten potential disease-related targets of arteannuin predicted by PharmMapper

\begin{tabular}{clcl}
\hline PDB ID & Name & Fit Score & Disease \\
\hline 1R1H & Neprilysin & 3.635 & Acute lymphocytic leukaemia \\
1RLB & Transthyretin & 3.548 & Amyloidosis type 1 \\
1RBP & Retinol-binding protein 4 & 3.511 & Retinol-binding protein deficiency \\
1R55 & ADAM33 & 3.418 & Asthma \\
1DIC & Complement factor D & 3.381 & Complement factor D deficiency \\
1GS4 & Androgen receptor & 3.303 & Androgenetic alopecia \\
1S9J & Dual specificity mitogen-activated protein kinase 1 & 3.294 & Cardiofaciocutaneous syndrome \\
1G48 & Carbonic anhydrase 2 & 3.259 & Autosomal recessive osteopetrosis type 3 \\
1TFG & Transforming growth factor beta-2 & 3.242 & Peters anomaly \\
1PMN & Mitogen-activated protein kinase 10 & 3.114 & Epileptic encephalopathy \\
\hline
\end{tabular}


TABLE II - Results of the arteannuin-neprilysin interactome using DRAR-CPI and DDI-CPI

\begin{tabular}{ccccccc}
\hline \multicolumn{4}{c}{ DRAR-CPI } & & \multicolumn{2}{c}{ DDI-CPI } \\
\cline { 1 - 3 } \cline { 5 - 6 } PDB ID & Affinity Score & Z-score & Z'-score & & PDB ID & Docking Score \\
\hline 1R1H & -22.9126 & 0.58101 & 2.3121 & & 1DMT & $-7.5(\mathrm{kcal} / \mathrm{mol})$ \\
\hline
\end{tabular}

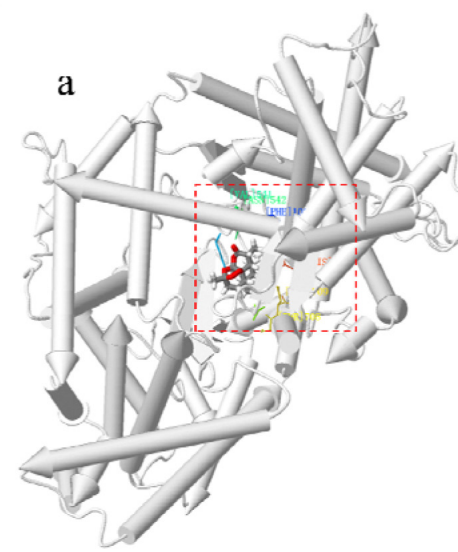

b

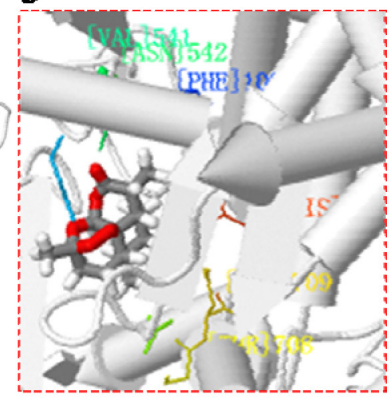

FIGURE 3 - Binding pattern of arteannuin and neprilysin (1DMT) obtained using the DRAR-CPI sever. (a) Overview. (b) Detailed view. The protein chain is shown in rocket representation, ligands are shown in stock representation, and key residues are coloured and shown in line representation.

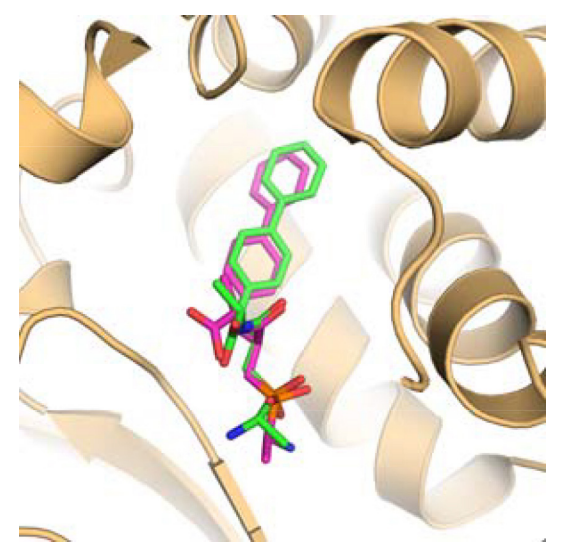

FIGURE 4 - Superimposition of the redocking pose (magenta) and the ligand pose in the co-crystal structure (green). The receptor is shown in cartoon representation and is coloured light orange.

Using AutoDock Vina in PyRx 0.8, the binding energy of the lowest state of the arteannuin-neprilysin complex was calculated as $-5.7 \mathrm{kcal} / \mathrm{mol}$ (Table III). As shown in Figure 5, arteannuin formed one hydrogen bond with residue His711. In addition, residues Phe106 and Trp693 formed hydrophobic contacts with arteannuin. Comparison of the putative binding model of arteannuin with the experimentally determined binding model from the co-crystal structure (PDB code $1 \mathrm{R} 1 \mathrm{H}$ ) is shown in Figure 5.

\section{DISCUSSION}

The potential for computational drug repositioning is great since this systematic process can incorporate prioritisation information that can accelerate the drug discovery process (Hurle et al., 2013). Reverse or inverse docking has proven to be a powerful tool for drug repositioning and drug rescue (Kharkar, Warrier, Gaud, 2014). In this study, neprilysin was found to be the top disease-related target of arteannuin using the reverse docking server PharmMapper (Table I). A pharmacophore is a molecular framework that includes the essential features responsible for the biological activity of a drug (Yang, 2010). The pharmacophore model generated in this study is in good agreement with the molecular features of arteannuin (Figure 2), which further supports the PharmMapper results.

A chemical-protein interactome is an estimate of the strength of interactions between a matrix of drugs and multiple human proteins, and adopting a chemicalprotein interactome approach can identify unexpected drug-protein interactions from computational strategies such as docking, chemical structure comparison, and text-mining (Yang et al., 2011). In the DRAR-CPI results of the present study, targets with a Z'-score less than -0.5 were treated as favourable and those with a Z'-score $>0.5$ as unfavourable (Luo et al., 2011; Chen, 2014). Based on the Z'-score from DRAR-CPI and the docking score from DDI-CPI (Table II), neprilysin is a potential candidate target of arteannuin that warrants further investigation.

TABLE III - Binding site coordinates for the pose with the lowest binding energy

\begin{tabular}{cccc}
\hline PDB ID & Name & Centre $(\mathbf{x} \times \mathbf{y} \times \mathbf{z})$ & Binding Energy $(\mathbf{k c a l} / \mathbf{m o l})$ \\
\hline 1R1H & Neprilysin & $31.27 \times 44.58 \times 34.62$ & -5.7 \\
\hline
\end{tabular}




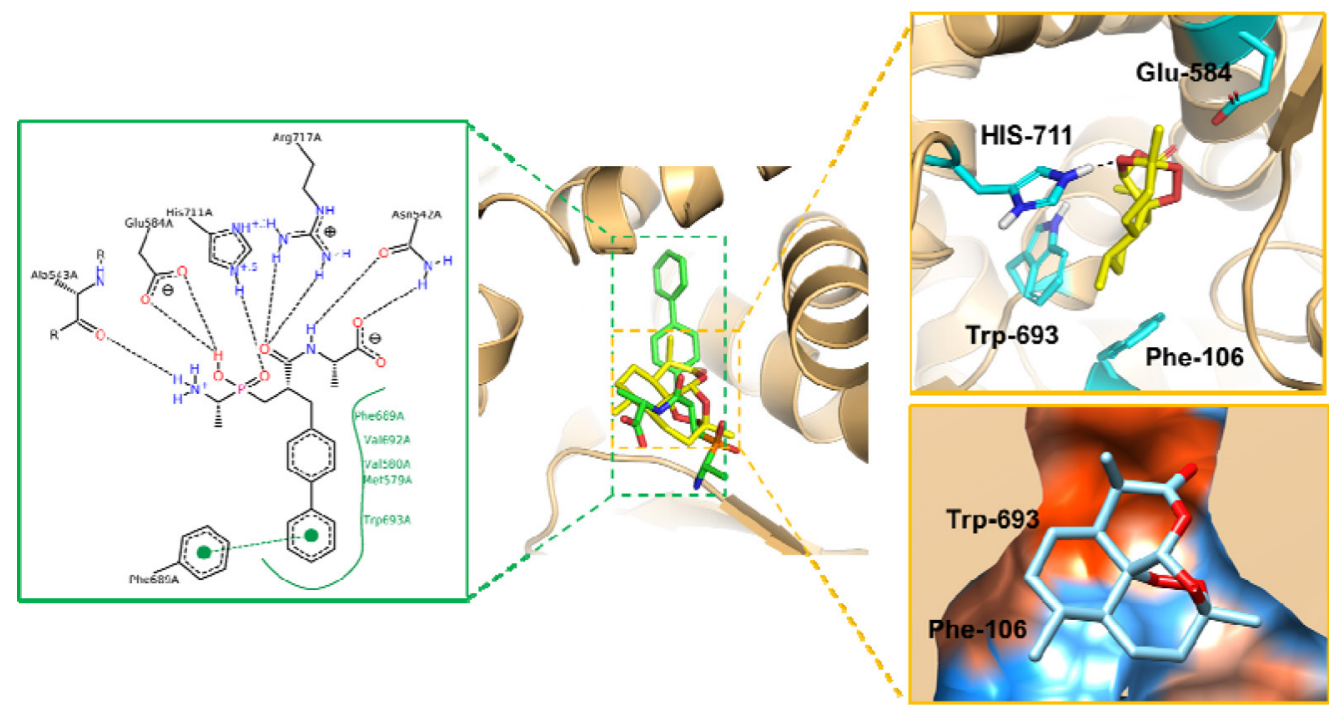

FIGURE 5 - Comparison of the putative binding mode of arteannuin (yellow) and the experimental BIR ligand-binding mode observed in the co-crystal structure (green; PDB code 1R1H). The receptor is shown in light orange cartoon representation. Black dashed lines indicate hydrogen bonds. The green solid line represents hydrophobic interactions and green dashed lines show $\pi-\pi$ interactions. Hydrophobic and hydrophilic residues are represented by orange red surface and dodger blue surface, respectively (in right bottom panel).

Importantly, the DRAR-CPI and DDI-CPI results were in good agreement with the PharmMapper results (Table I).

Protein-ligand interactions can provide an indepth understanding of complex processes at a structural level (Chen, 2014). As shown in the published proteinligand structure used in this study, the original ligand BIR interacts with neprilysin $(1 \mathrm{R} 1 \mathrm{H})$ via numerous complicated interactions that include hydrogen bonds with Ala543, Glu584, His711, Arg717, and Asn542; hydrophobic interactions with Phe689, Val692, Val580, Met579, and Trp693; and $\pi$-cation interactions with Phe689 (Oefner et al., 2004). Like the original ligand BIR, arteannuin may also bind many of these key residues (Figure 3 and 5). Residues His583, His587, and Glu646 are involved in zinc coordination, and may also participate in monodentate or bidentate recognition, depending on the ligand (Oefner et al., 2004). Arg717 participates in a salt bridge with Asp650 (Oefner et al., 2004), and Phe106, Ile558, Phe563, Met579, Val580, Val692, and Trp693 form a deep lipophilic cavity (Oefner et al., 2004). The pharmacological functions of arteannuin may therefore be dependent on these interactions.

To gain further insight into the binding mechanism between arteannuin and neprilysin, the putative arteannuin binding pose was superimposed onto the ligand in the cocrystal structure (PDB code 1R1H). As seen in Figure 5, compared with the ligand in $1 \mathrm{R} 1 \mathrm{H}$, arteannuin occupies less space within the binding pocket of neprilysin due to its smaller size. Interestingly, we noted that the polar groups of both ligands are located at the bottom of the binding site, which indicated that hydrophilic protein residues may be more important for binding. Indeed, there is a complex hydrogen bonding network between the native ligand and the neprilysin protein, whereas arteannuin only forms one hydrogen bond, with the side chain of His-711, in the highest scoring docking pose. Conversely, the residues at the top of the binding pocket are more hydrophobic and form multiple hydrophobic interactions with both ligands.

As shown in Table I, neprilysin (also known as CD10) is an important cell surface marker in the diagnosis of human acute lymphocytic leukaemia. It is a ubiquitous transmembrane and circulating protease that cleaves a broad range of substrates (Oefner et al., 2004; Vodovar et al., 2015). First identified in leukaemia as a tumour-specific antigen (common acute lymphoblastic leukaemia antigen), CD10 is now widely used in cancer diagnosis (Maguer-Satta, Besancon, Bachelard-Cascales, 2011). Arteannuin and its analogues are reported to act as effective anticancer chemotherapeutic agents in Molt-4 human leukaemia cells (Singh, Lai, 2004; Ohgami et al., 2010; Singh et al., 2011). The results of the present study suggest that the anticancer activity of artemisinin in acute lymphocytic leukaemia may be related to the targeting of neprilysin.

Neprilysin is also known as a neutral endopeptide that has been implicated in the catabolism of amyloid beta peptides in the brain, which has attracted considerable attention, particularly as a potential therapeutic target for 
Alzheimer's disease (Hersh, Rodgers, 2008). Arteannuin extenuates amyloidogenesis and neuroinflammation in APPswe/PS1De9 transgenic mice via inhibition of nuclear factor- $\mathrm{kB}$ and NLRP3 inflammasome activation (Shi et al., 2013). Arteannuin may therefore be a potential treatment for Alzheimer's disease via the targeting of neprilysin.

\section{CONCLUSION}

In summary, reverse docking revealed neprilysin as a potential target of arteannuin, and the results were assessed by a chemical-protein interactome approach using DRARCPI, DDI-CPI, and AutoDock Vina. Despite the need for further experimental verification, this study provides guidance for future pharmacological investigations into novel clinical applications for arteannuin, and may have implications for the treatment of acute lymphocytic leukaemia or Alzheimer's disease.

\section{ACKNOWLEDGEMENTS}

This work was supported by the Zhejiang Provincial Natural Science Foundation of China (LY15H280009), the Ningbo Municipal Natural Science Foundation (2015A610280), and the Administration of Traditional Chinese Medicine of Zhejiang Province (2014ZB110).

\section{REFERENCES}

AERI, L.; KYOUNGYEUL, L.; DONGSUP, K. Using reverse docking for target identification and its applications for drug discovery. Expert Opin. Drug Discov., v.11, n.7, p.707-715, 2016.

CHEN, S.J. A potential target of Tanshinone IIA for acute promyelocytic leukemia revealed by inverse docking and drug repurposing. Asian Pac. J. Cancer Prev., v.15, n.10, p.4301-4305, 2014.

CHEN, S.J.; REN, J.L. Identification of a potential anticancer target of danshensu by inverse docking. Asian Pac. J. Cancer Prev., v.15, n.1, p. 111-6, 2014.

CHEN, Y.Z., ZHI, D.G. Ligand-protein inverse docking and its potential use in the computer search of protein targets of a small molecule. Proteins, v.43, n.2, p.217-226, 2001.

HERSH, L.B.; RODGERS, D.W. Neprilysin and amyloid beta peptide degradation. Curr. Alzheimer Res., v.5, n.2, p.22531, 2008.
HUANG, C.; BA, Q.; YUE, Q.; LI, J.; CHU, R.; WANG, H. Artemisinin rewires the protein interaction network in cancer cells: network analysis, pathway identification and target prediction. Mol. Biosyst., v. 9, n.12, p. 3091-3100, 2013.

HURLE, M.R.; YANG, L.; XIE, Q.; RAJPAL, D.K.; SANSEAU, P.; AGARWAL, P. Computational drug repositioning: from data to therapeutics. Clin. Pharmacol. Ther., v.93, n.4, p. 335-41, 2013.

KHARKAR, P.S.; WARRIERR, S.; GAUD, S. Reverse docking: a powerful tool for drug repositioning and drug rescue. Future Med. Chem., v.6, n.3, p.333-42, 2014.

KUMAR, S.; JENA, L.; MOHOD, K.; DAF, S.; VARMA, A.K. Virtual screening for potential inhibitors of high-risk human papillomavirus 16 E6 protein. Interdiscip. Sci., v.7, n.2, p136-142, 2015.

LIU, X.; OUYANG, S.; YU, B.; LIU, Y.; HUANG, K.; GONG, J.; ZHENG, S.; LI, Z.; LI, H.; JIANG, H. PharmMapper server: a web server for potential drug target identification using pharmacophore mapping approach. Nucleic Acids Res., v.38, p.W609-14, 2010.

LUO, H.; CHEN, J.; SHI, L.; MIKAILOV, M.; ZHU, H.; WANG, K.; HE, L.; YANG, L. DRAR-CPI: a server for identifying drug repositioning potential and adverse drug reactions via the chemical-protein interactome. Nucleic Acids Res., v.39, p.W492-8, 2011.

LUO, H.; ZHANG, P.; HUANG, H.; HUANG, J.; KAO, E.; SHI, L.; HE, L.; YANG, L. DDI-CPI, a server that predicts drug-drug interactions through implementing the chemicalprotein interactome. Nucleic Acids Res., v.42, p.W46-52, 2014.

MAGUER-SATTA, V.; BESANCONE, R.; BACHELARDCASCALES, E. Concise review: neutral endopeptidase (CD10): a multifaceted environment actor in stem cells, physiological mechanisms, and cancer. Stem Cells, v.29, n.3, p. 389-96, 2011.

MORRIS, G.M.; HUEY, R.; LINDSTROM, W.; SANNER, M.F.; BELEW, R.K.; GOODSELL, D.S.; OLSON, A.J. Autodock4 and AutoDockTools4: automated docking with selective receptor flexibility. J. Comp. Chem., v.30, n.16, p. 2785-2791, 2009. 
O’NEILL, P.M.; BARTON, V.E.; WARD, S.A. The molecular mechanism of action of artemisinin--the debate continues. Molecules, v.15, n.3, p.1705-21, 2010.

OEFNER, C.; ROQUES, B.P.; FOURNIE-ZALUSKIG, M.C.; DALE, G.E. Structural analysis of neprilysin with various specific and potent inhibitors. Acta Crystallogr. D.: Biol. Crystallogr., v.60, pt. 2, p.392-6, 2004.

OHGAMI, Y.; ELSTAD C.A.; CHUNG, E.; SHIRACHI, D.Y.; QUOCKH R.M.; LAI, H.C. Effect of hyperbaric oxygen on the anticancer effect of artemisinin on molt-4 human leukemia cells. Anticancer Res., v.30, n.11, p.4467-70, 2010 .

QIU, J.X.; ZHOU, Z.W.; HE, Z.X.; ZHAO, R.J.; ZHANG, X.; YANG, L.; ZHOU, S.F.; MAO, Z.F. Plumbagin elicits differential proteomic responses mainly involving cell cycle, apoptosis, autophagy, and epithelial-to-mesenchymal transition pathways in human prostate cancer PC-3 and DU145 cells. Drug Des. Devel. Ther., v.9, p.349-417, 2015.

SHI, J.Q.; ZHANG, C.C.; SUN, X.L.; CHENG, X.X.; WANG, J.B.; ZHANG, Y.D.; XU, J.; ZOU, H.Q. Antimalarial drug artemisinin extenuates amyloidogenesis and neuroinflammation in APPswe/PS1dE9 transgenic mice via inhibition of nuclear factor-kappaB and NLRP3 inflammasome activation. CNS Neurosci. Ther, v.19, n.4, p.262-8, 2013.

SINGH, N.P.; FERREIRA, J.F.; PARK, J.S.; LAI, H.C. Cytotoxicity of ethanolic extracts of Artemisia annua to Molt-4 human leukemia cells. Planta Med., v.77, n.16, p.1788-93, 2011.

SINGH, N.P.; LAI, H.C. Artemisinin induces apoptosis in human cancer cells. Anticancer Res., v. 24, n. 4, p. $2277-$ 80, 2004.

TROTT, O.; OLSON, A.J. AutoDock Vina: improving the speed and accuracy of docking with a new scoring function, efficient optimization, and multithreading. J. Comput. Chem., v. 31, n. 2, p. 455-61, 2010.
TU, Y. The discovery of artemisinin (qinghaosu) and gifts from Chinese medicine. Nat. Med., v.17, n.10, p.1217-20, 2011.

USHA, T.; GOYAL, A.K.; LUBNA, S.; PRASHANTH, H.; MOHAN, T.M.; PANDE, V.; MIDDHA, S.K. Identification of anti-cancer targets of eco-friendly waste Punica granatum peel by dual reverse virtual screening and binding analysis. Asian Pac. J. Cancer Prev., v.15, n.23, p.10345-50, 2014.

VODOVAR, N.; PAQUET, C.; MEBAZAA, A.; LAUNAY, J.M.; HUGON, J.; COHEN-SOLAL, A. Neprilysin, cardiovascular, and Alzheimer's diseases: the therapeutic split? Eur. Heart J., v.36, n.15, p.902-905, 2015.

VOLKAMER, A.; RAREY, M. Exploiting structural information for drug-target assessment. Future Med. Chem., v.6, n.3, p.319-31, 2014.

WHITE, N.J. Qinghaosu (artemisinin): the price of success. Science, v.320, n.5874, p.330-4, 2008.

YANG, L.; WANG, K.J.; WANG, L.S.; JEGGA, A.G.; QIN, S.Y.; HE, G.; CHEN, J.; XIAO, Y.; HE, L. Chemical-protein interactome and its application in off-target identification. Interdiscip. Sci., v.3, n.1, p.22-30, 2011.

YANG, S.Y. Pharmacophore modeling and applications in drug discovery: challenges and recent advances. Drug Discov. Today, v.15, n.11-12, p.444-50, 2010.

YE, X.Y.; LING, Q.Z.; CHEN, S.J. Identification of a potential target of capsaicin by computatioanl target fishing. Evid. Based Complement Alternat. Med., v.2015, p.983951, 2015.

YUAN, D.P.; LONG, J.; LU, Y.; LIN, J.; TONG, L. The forecast of anticancer targets of cryptotanshinone based on reverse pharmacophore-based screening technology. Chin. J. Nat. Med., v.12, n.6, p.443-8, 2014.

Received for publication on $08^{\text {th }}$ May 2016 ? Accepted for publication on $19^{\text {th }}$ December 2016 